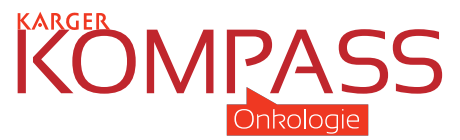

\author{
29. Deutscher Hautkrebskongress \\ 11.-14. September 2019 \\ Ludwigshafen, Deutschland \\ www.ado-kongress.de
}

29. Deutscher Hautkrebskongress ADO-Jahrestagung 11.-14. September 2019 Ludwigshafen
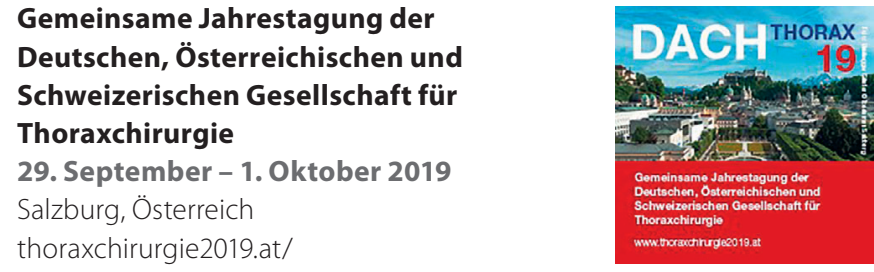

\author{
52. Jahrestagung der Deutschen \\ Gesellschaft für Transfusionsmedizin \\ und Immunhämatologie e.V. (DGTI) \\ 18.-20. September 2019 \\ Mannheim, Deutschland \\ https://www.dgti-kongress.de/
}

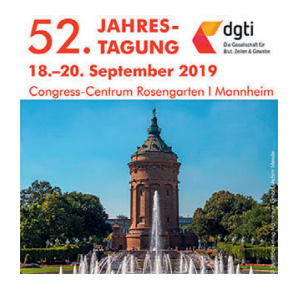

Jahrestagung der Deutschen, Österreichischen und Schweizerischen Gesellschaften für Hämatologie und Medizinische Onkologie 11.-14.Oktober 2019 Berlin, Deutschland

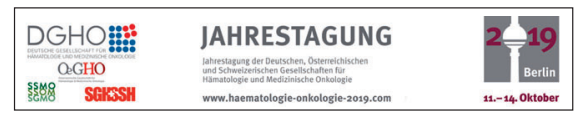
www.haematologie-onkologie-2019.com

\section{SEPTEMBER 2019}

ESID 2019 - Focused Meeting:

Malignancy and PID

18.-21. September 2019

Brüssel, Belgien

https://esidmeeting.org/scientific-programme/ why-malignancy-and-pid/

\section{Jahrestagung der Arbeitsgemein-} schaft für Psychoonkologie (PSO)

19.-21. September 2019

Düsseldorf, Deutschland

https://www.pso-jahrestagungen.de

Onkologie - Beruf oder Berufung? 5. Autumn School für Medizinstudierende 2019

23.-25. September 2019

Berlin, Deutschland

www.ng-akademie.de/details/onkologieberuf-oder-berufung-5-autumn-school-fuermedizinstudierende-2019.html

\section{Post ICML Meeting / Deutsches}

Lymphomforum 2019

27.-28. September 2019

Göttingen, Deutschland

https://www.dgho.de/veranstaltungen/ veranstaltungen/kalender_2019/post-icmlmeeting-deutsches-lymphomforum-2019

\section{ESMO 2019 Congress}

27. September bis 01. Oktober 2019

Barcelona, Spanien

https://www.dgho.de/veranstaltungen/ veranstaltungen/kalender_2019/esmo-2019congress

\section{OKTOBER 2019}

Interdisziplinäre ESMO-Nachlese 16. Oktober 2019

Berlin, Deutschland

https://www.dgho.de/veranstaltungen/

veranstaltungen/kalender_2019/

interdisziplinaere-esmo-nachlese

\section{Franken Fortbildung Frauen-} gesundheit

25.-26. Oktober 2019

Würzburg, Deutschland

https://www.if-kongress.de/veranstaltungen/

\section{NOVEMBER 2019}

\section{ENDOCLUBNORD 2019}

\section{1.-2. November 2019}

Hamburg, Deutschland

www.endoclubnord.de

\section{Interdisziplinäres Prostatakarzinom- Symposium \\ 07. November 2019 \\ St. Gallen, Schweiz \\ https://www.oncoconferences.ch/PRO2019}

10. MDS Colloquium featuring 7. EMSCO Meeting und die 1. MDS Academy 08.-09. November 2019

Leipzig, Deutschland

http://mds-colloquium.com/ 19th Conference of the International Society of Geriatric Oncology

14.-16. November 2019

Geneva, Schweiz

https://www.siogconference.org/

\section{AIO-Herbstkongress - Update}

Medical Oncology

21.-23. November 2019

Berlin, Deutschland

http://www.aio-herbstkongress.de/

\section{Ostsee-Seminar}

29.-30. November 2019

Kiel, Deutschland

https://www.ff-kongress.de/veranstaltungen/

\section{DEZEMBER 2019}

\section{Wintersymposium}

14. Dezember 2019

München, Deutschland

https://www.wintersymposium-muenchen.de

\section{FEBRUAR 2020}

\section{Deutscher Krebskongress \\ 19.-22. Februar 2020 \\ Berlin, Deutschland}

8th European Conference on Tobacco or Health - ECToH 2020

19.-22. Februar 2020

Berlin, Deutschland

www.ng-akademie.de/details/ECToH2020.html

\title{
KARGER
}

(C) 2019 S. Karger GmbH, Freiburg 